\title{
The Correlations of Disease Activity, Socioeconomic Status, Quality of Life, and Depression/Anxiety in Chinese Patients with Systemic Lupus Erythematosus
}

\author{
Biyu Shen, ${ }^{1,2}$ Wei Tan, ${ }^{3}$ Guijuan Feng, ${ }^{3}$ Yan He, ${ }^{1}$ Jinwei Liu, ${ }^{1}$ Weijun Chen,, \\ Xiaoqin Huang, ${ }^{1}$ Zhanyun $\mathrm{Da}^{3}{ }^{\mathrm{Xujuan}} \mathrm{Xu},{ }^{2}$ Hong Liu, ${ }^{3}$ and Zhifeng $\mathrm{Gu}^{3}$ \\ ${ }^{1}$ Department of Nursing, The Second Affiliated Hospital of Nantong University, Nantong, Jiangsu 226001, China \\ ${ }^{2}$ School of Nursing, Nantong University, Nantong, Jiangsu 226001, China \\ ${ }^{3}$ Department of Rheumatology, The Affiliated Hospital of Nantong University, Nantong, Jiangsu 226001, China
}

Correspondence should be addressed to Zhifeng Gu; guzhifeng@126.com

Received 14 April 2013; Accepted 9 June 2013

Academic Editor: Guixiu Shi

Copyright ( $\odot 2013$ Biyu Shen et al. This is an open access article distributed under the Creative Commons Attribution License, which permits unrestricted use, distribution, and reproduction in any medium, provided the original work is properly cited.

\begin{abstract}
The prevalence of psychological problems is frequent in systemic lupus erythematosus (SLE) patients and appears to be increasing. The current study investigated the relationship among disease parameters, quality of life, and the psychological status in Chinese patients with SLE. A self-report survey design was administered to 170 SLE patients and 210 healthy individuals using the SelfRating Anxiety Scale, the Self-Rating Depression Scale, and the Short Form 36 health survey (SF-36). Our results showed that $20.3 \%$ SLE patients had anxiety, and 32.9\% had depression, which were significantly higher than the control group $(7.1 \%, 14.3 \%$, resp.). And there were significant correlations among socioeconomic status (SES), disease activity, and anxiety/depression in SLE patients. Meanwhile, SF-36 analysis results revealed that VT, PF, and RP scales were the most powerful predictors of anxiety of SLE patients, and SLEDAI, VT, PF, SF, and RE domains were significantly accounted for anxiety. In summary, there were significant relationships among disease parameters, quality of life, and anxiety/depression in Chinese SLE patients. Therefore, it is necessary to have psychiatric and psychological evaluations and formulate an integrated approach for managing mental health in Chinese lupus patients, especially those who have high disease activity, low SES, and poor quality of life.
\end{abstract}

\section{Introduction}

Systemic lupus erythematosus (SLE) is a chronic inflammatory autoimmune disease that may affect multiple organ systems, including the central nervous system (CNS) [1]. Psychiatric symptoms are present in the majority of patients with SLE, among which major depression is the most common psychiatric manifestations [2]. Depression presented in $11 \%-39 \%$ of patients may be the initial symptom before the diagnosis of SLE [3]. It was reported that there were 4 times higher prevalence of depression in SLE compared to a matched, non-SLE population. In addition, anxiety is quite common in SLE patients, often as a reaction to the illness. Ainiala and colleagues have reported that the anxiety disorders were twice as prevalent among SLE patients as compared to the controls [4].

Even though SLE presents accompany with a wide variety of treatable psychiatric symptoms, such as depression and anxiety, they rarely seek and receive adequate treatment [5]. Overlooking anxiety and depression may have severe consequences for the patients, such as increased incidence of cardiovascular disease [6], myocardial infarction [7], suicidal ideation $[8,9]$ and death [10], decreased quality of life [11, 12], disability, and the loss of employment. Anyone, in turn, can worsen anxiety and depression symptoms [5].

The pathogenesis of psychiatric symptoms in lupus is still not well understood, but in which genetic and environmental factors may play a pivotal role. Depression and anxiety 
may also be present as a reaction to a serious recurring, painful illness, which is associated with visible symptoms such as insomnia, fatigue, and limited functioning $[13,14]$. Socioeconomic status (SES) is broadly employed in health research, signaling the importance of socioeconomic factors for health outcomes. Low SES is generally associated with high psychiatric morbidity, depression [15], and mortality [16]. Whether depression and anxiety are associated with lupus activity remains debatable.

There are several studies focus on psychological problems in China lupus patients. A study from Hong Kong has found that anxiety disorder was present in $22 \%$ SLE patients, and 18.2\% had depression [17]. A study from Anhui medical university has reported that the prevalence of depression was $59.3 \%$ and correlated with suicidal ideation in SLE patients [8]. But there are few studies that focus on disease parameters, quality of life, and depression/anxiety in SLE patients from China mainland.

Thus, the aim of this study was to examine the relationship among disease parameters, quality of life, and the psychological status in Chinese patients with SLE. Moreover, we wished to ascertain the possible risks of anxiety and depression.

\section{Patients and Methods}

2.1. Participants. SLE patients were recruited from Affiliated Hospital of Nantong University between January 2010 and July 2011. A total of 170 SLE patients and 210 healthy individuals were consecutively invited to participate in a singlecenter cross-sectional study. Healthy individuals were used as the control group. All patients fulfilled the 1997 American College of Rheumatology (ACR) revised criteria for the classification of SLE. Patients were excluded based on the following: (1) they did not complete questionnaire; (2) they had comorbidities (e.g., serious infections or cardiac, respiratory, gastrointestinal, neurological, or endocrine diseases) that could influence SLE activity. Control subjects were excluded if they exhibited current or history of other systemic diseases or psychiatric disorders. This study was approved by the Ethics Committee of Affiliated Hospital of Nantong University, and written informed consent was obtained from all participants.

2.2. Measures of Clinical Variables. The Systemic Lupus Erythematosus Disease Activity Index (SLEDAI) was used to measure disease activity [18].

2.3. The Revised Self-Rating Anxiety Scale (SAS) [19]. SAS was used to evaluate the level of anxiety-related symptoms during the week prior to the survey. This self-administered test has 20 questions, with 15 items reflecting increasing anxiety levels and 5 questions reflecting decreasing anxiety levels. Each question was scored on a scale from 1 to 4 (rarely, sometimes, frequently, and always). The scores ranged between 20 and 80: scores greater than 70 suggest severe anxious symptoms, scores between 60 and 69 indicate moderate to marked anxiety, scores between 50 and 59 suggest minimal to mild anxiety, and scores less than 50 indicate no anxious symptoms.

2.4. The Revised Self-Rating Depression Scale (SDS) [20]. SDS is a 20-item questionnaire designed to assess mood symptoms over the past week (e.g., "I feel downhearted, blue and sad"). Each item is scored on a Likert scale ranging from 1 to 4; scores greater than 70 suggest severe depressive symptoms, scores between 60 and 69 indicate moderate to marked depression, scores between 50 and 59 suggest minimal to mild depression, and scores less than 50 indicate no depressive symptoms.

2.5. Measure of the Quality of Life [21]. The patient's general health status was measured using the Short Form- (SF-) 36 questionnaire, which measured eight multi-item dimensions: physical functioning (PF, 10 items); role limitations due to physical problems (RP, four items); role limitations due to emotional problems (RE, three items); social functioning (SF, two items); mental health ( $\mathrm{MH}$, five items); energy/vitality (VT, four items); body pain (BP, two items); and general health perception ( $\mathrm{GH}$, five items). For each dimension, item scores were coded, summed, and transformed on a scale from 0 (worst possible health state measured by the questionnaire) to 100 (best possible health state).

2.6. Statistical Analysis. The data were expressed as means \pm SDs for continuous variables and as frequencies (\%) for categorical variables. The Statistical Package for SPSS 18.0 was used for all data management and analyses. Descriptive analyses were performed to investigate the participants' characteristics. Student's $t$-test was used in independent groups for parametric variables, and the Spearman's correlation analysis was used to assess the correlation between parametric variables. Stepwise regression analyses were conducted for SAS and SDS scores separately to explore the significant predictors of dimorphic concerns. We considered $P<0.01$ and $P<0.001$ to be highly statistically significant and $P<$ 0.05 to be statistically significant.

\section{Results}

3.1. Sample Characteristics. 12 SLE patients and 14 healthy individuals did not complete the questionnaire, resulting in the enrollment of 158 SLE patients (14 males and 144 females) and 196 healthy individuals (20 males and 176 females) in the current study. Table 1 showed their demographic data, medical and psychological variables. There was no significant difference in the ages, genders, marital status, education, work status, income/person, and menstrual history between the SLE patients and the controls. The SAS and SDS scores were significantly higher in the SLE group compared to the control group. According to the cut-off scores, anxiety disorder was present in 32/158 (20.3\%), and 52/158 (32.9\%) had depression, which were significantly higher than the healthy group $((14 / 196,7.1 \%)$ and $(28 / 196,14.3 \%)$, resp. $)(P<$ $0.01)$. As shown in Table 2 , the scores of all the 8 scales were lower in SLE patients compared with healthy individuals. 
TABLE 1: Demographic, psychological, and disease characteristics in SLE patients and controls.

\begin{tabular}{lccc}
\hline Variables & $\begin{array}{c}\text { SLE patients } \\
(N=158)\end{array}$ & $\begin{array}{c}\text { Control subjects } \\
(N=196)\end{array}$ & $P$ \\
\hline Female gender $^{\mathrm{a}}$ & $144(91.2)$ & $176(89.8)$ & 0.76 \\
Age, years $^{\mathrm{b}}$ & $32.9 \pm 10.2$ & $35.0 \pm 11.4$ & 0.20 \\
SAS $(\geq 50)^{\mathrm{a}}$ & $32(20.3)$ & $14(7.1)$ & $<0.01$ \\
SDS $(\geq 53)^{\mathrm{a}}$ & $52(32.9)$ & $28(14.3)$ & 0.003 \\
SLEDAI & $11.8 \pm 9.5$ & & \\
Marital status & & & \\
$\quad$ Single & $32(20.3)$ & $56(18.6)$ & 0.20 \\
$\quad$ Married & $126(79.7)$ & $140(71.4)$ & \\
Education & & & \\
$\quad<9$ years & & $96(49.0)$ & 0.47 \\
$\quad \geq 9$ years & $72(45.6)$ & $100(51.0)$ & \\
Work status & & & \\
$\quad$ Working & $30(19.0)$ & $44(22.5)$ & 0.57 \\
$\quad$ Unemployed & $128(81.0)$ & $152(77.5)$ & \\
Income/person & & & \\
$\quad \leq 2000$ yuan & $100(63.3)$ & $118(60.2)$ & 0.68 \\
$\quad>2000$ yuan & $58(36.7)$ & $78(39.8)$ & \\
Menstrual history & & & \\
$\quad$ Normal & $96(66.7)$ & $102(58.0)$ & \\
$\quad$ Abnormal & $48(33.3)$ & $74(42.0)$ & \\
\hline
\end{tabular}

${ }^{a}$ Mean \pm SD. ${ }^{b}$ Number (percentage).

SAS: revised Self-Rating Anxiety Scale; SDS: revised Self-Rating Depression Scale; SLEDAI: Systemic Lupus Erythematosus Disease Activity Index.

TABLE 2: Correlations between psychological scores, disease parameters, and quality of life in SLE patients.

\begin{tabular}{lcccc}
\hline Variables & \multicolumn{2}{c}{ SAS } & \multicolumn{2}{c}{ SDS } \\
& $r$ & $P$ & $r$ & $P$ \\
\hline Domains of SF-36 & & & & \\
PCS & -0.53 & $<0.0001$ & -0.53 & $<0.0001$ \\
MCS & -0.68 & $<0.0001$ & -0.73 & $<0.0001$ \\
PF & -0.49 & $<0.0001$ & -0.54 & $<0.0001$ \\
RP & -0.55 & $<0.0001$ & -0.52 & $<0.0001$ \\
BP & -0.05 & 0.66 & 0.05 & 0.65 \\
GH & -0.36 & 0.001 & -0.37 & 0.0009 \\
VT & -0.4 & 0.0003 & -0.43 & 0.0001 \\
SF & -0.49 & $<0.0001$ & -0.57 & $<0.0001$ \\
RE & -0.63 & $<0.0001$ & -0.64 & $<0.0001$ \\
MH & -0.31 & 0.005 & 0.34 & 0.003 \\
\hline
\end{tabular}

SAS: revised Self-Rating Anxiety Scale; SDS: revised Self-Rating Depression Scale; PCS: physical components summary; MCS: mental components summary; PF: physical functioning; RP: role limitations due to physical problems; RE: role limitations due to emotional problems; SF: social functioning; MH: mental health; VT: energy/vitality; BP: body pain; GH: general health perception.

There was significant difference between physical functioning $(\mathrm{PF})$, role limitations due to physical problems (RP), role limitations due to emotional problems (RE), mental health
$(\mathrm{MH})$, and energy/vitality (VT) in SLE and control group $(P<0.05)$.

3.2. Correlations between Psychological Scores, Disease Parameters, and Quality of Life in SLE Patients. Previous studies have shown that low socioeconomic factors (SES) were generally associated with high psychiatric morbidity, depression, and anxiety [22]. As show in Table 3, we have found that there were significant correlations between SES (low education, work status, and income) and anxiety/depression in SLE patients. In addition, gender and menstrual history were some examples of depression risk factors. There was a significant positive correlation between anxiety/depression severity (assessed using SAS/SDS score) and disease activity (SLEDAI score). Previous studies have found that impaired quality of life and functional disability were independent risk factors of psychological disorders [12]. In the present study, we found that all the 8 scales of SF-36 domains and PCS/MCS were significantly correlated with SAS and SDS scores except body pain $(\mathrm{BP})$ scale $(P<0.05)$.

3.3. Stepwise Regression Analysis for Anxiety and Depression. Multiple stepwise regression analysis revealed that VT, PF, and RP scales of SF-36 were the most powerful predictors of anxiety of SLE patients (Table 4). Meanwhile, SLEDAI, VT, PF, SF, and RE domains of SF-36 were significantly accounted for anxiety (Table 5).

\section{Discussion}

The present study confirmed that Chinese SLE patients were more likely to suffer from anxiety and depression than healthy individuals. Psychological problems significantly correlated with SES, disease status, and quality of life. SLE patients with anxiety and depression were in low SES and had worse disease status, lower quality of life. Among the assessed parameters, VT, PF, and RP scales of SF-36 were major contributors to anxiety in SLE patients, while disease activity and VT, PF, SF, and RE domains of SF-36 contributed to depression.

SES is broadly employed in health research, signaling the importance of socioeconomic factors for health outcomes. Previous study has found that poorer coping styles, ongoing life events, stress exposure, and weaker social support were some examples of depression risk factors that were more prevalent in lower SES groups [23]. Regarding the direction of the association for SES and depression, results more consistently supported the idea that causation (low SES increases risk of depression) outweighed selection (depression hinders social mobility), although both directions may operate simultaneously [17]. It is well known that SES is multifactor. Occupation $[5,24,25]$, education, and income [26] were frequently used as measures of SES. With notable exceptions, there were significant relationships between anxiety/depression and SES [5]. There was a substantial body of research linking SES, anxiety/depression, and SLE. Waheed et al. found that educational qualification had significant association with anxiety and depression. Marital status, gender, economic activity, and monthly family income had no 
TABLE 3: Disease status and quality of life in the anxious and depressed subgroups.

\begin{tabular}{|c|c|c|c|c|c|c|}
\hline \multirow{2}{*}{ Variables } & \multicolumn{3}{|c|}{ SAS } & \multicolumn{3}{|c|}{ SDS } \\
\hline & $<50$ & $\geq 50$ & $P$ & $<53$ & $\geq 53$ & $P$ \\
\hline $\operatorname{Age}^{\mathrm{a}}$ & $32.3 \pm 10.3$ & $35.2 \pm 9.6$ & 0.32 & $32.4 \pm 10.6$ & $34.0 \pm 9.4$ & 0.51 \\
\hline \multicolumn{7}{|l|}{$\operatorname{Sex}^{b}$} \\
\hline Male & $6(9.5)$ & $1(6.3)$ & 0.68 & $7(13.2)$ & $0(0.0)$ & 0.05 \\
\hline Female & $57(90.5)$ & $15(92.7)$ & & $46(86.8)$ & $26(100.0)$ & \\
\hline BMI & $21.2 \pm 2.7$ & $21.1 \pm 2.9$ & 0.93 & $21.1 \pm 2.8$ & $21.4 \pm 2.6$ & 0.61 \\
\hline \multicolumn{7}{|l|}{ Marital status ${ }^{\mathrm{b}}$} \\
\hline Single & $15(23.8)$ & $1(6.3)$ & 0.25 & $14(26.4)$ & $2(7.7)$ & 0.11 \\
\hline Married & $48(76.2)$ & $15(93.7)$ & & $38(73.6)$ & $24(92.3)$ & \\
\hline \multicolumn{7}{|l|}{ Education $^{\mathrm{b}}$} \\
\hline$<9$ years & $29(46.0)$ & $14(87.5)$ & 0.003 & $23(43.4)$ & $20(76.9)$ & 0.005 \\
\hline$\geq 9$ years & $34(54.0)$ & $2(12.5)$ & & $30(56.6)$ & $6(23.1)$ & \\
\hline \multicolumn{7}{|l|}{ Work status ${ }^{\mathrm{b}}$} \\
\hline Working & $15(23.8)$ & $0(0)$ & 0.03 & $13(24.5)$ & $2(7.7)$ & 0.07 \\
\hline Unemployed & $48(76.2)$ & $16(100.0)$ & & $40(75.5)$ & $24(92.3)$ & \\
\hline \multicolumn{7}{|l|}{ Income/person ${ }^{\mathrm{b}}$} \\
\hline$\leq 2000$ yuan & $36(57.1)$ & $14(87.5)$ & 0.02 & $28(52.8)$ & $22(84.6)$ & 0.006 \\
\hline$>2000$ yuan & $27(42.9)$ & $2(12.5)$ & & $25(47.2)$ & $4(15.4)$ & \\
\hline \multicolumn{7}{|c|}{ Menstrual history ${ }^{\mathrm{b}}$} \\
\hline Normal & $39(68.4)$ & $9(60.0)$ & 0.54 & $36(79.2)$ & $12(46.2)$ & 0.006 \\
\hline Abnormal & $18(31.6)$ & $6(40.0)$ & & $10(20.8)$ & $14(53.8)$ & \\
\hline \multicolumn{7}{|c|}{ Years since diagnosis of SLE ${ }^{b}$} \\
\hline$<1$ & $11(17.5)$ & $2(12.5)$ & 0.85 & $10(18.9)$ & $3(11.5)$ & 0.63 \\
\hline $1-5$ & $31(49.2)$ & $9(56.3)$ & & $27(50.9)$ & $13(50.0)$ & \\
\hline$>5$ & $21(33.3)$ & $5(31.2)$ & & $16(30.2)$ & $10(38.5)$ & \\
\hline SLEDAI $^{\mathrm{a}}$ & $10.4 \pm 7.3$ & $17.1 \pm 14.7$ & 0.01 & $10.3 \pm 7.7$ & $14.8 \pm 12.1$ & 0.046 \\
\hline \multicolumn{7}{|c|}{ Domains of SF-36 } \\
\hline $\mathrm{PCS}^{\mathrm{a}}$ & $256.7 \pm 60.7$ & $158.1 \pm 51.7$ & $<0.0001$ & $264.0 \pm 58.6$ & $181.1 \pm 61.5$ & $<0.0001$ \\
\hline $\mathrm{MCS}^{\mathrm{a}}$ & $282.8 \pm 67.0$ & $161.7 \pm 60.1$ & $<0.0001$ & $295.1 \pm 56.9$ & $183.3 \pm 72.9$ & $<0.0001$ \\
\hline $\mathrm{PF}^{\mathrm{a}}$ & $86.8 \pm 14.1$ & $61.3 \pm 28.8$ & $<0.0001$ & $88.9 \pm 12.2$ & $66.9 \pm 26.1$ & $<0.0001$ \\
\hline $\mathrm{RP}^{\mathrm{a}}$ & $52.4 \pm 42.5$ & $4.7 \pm 13.6$ & 0.0001 & $57.1 \pm 43.1$ & $13.5 \pm 23.7$ & $<0.0001$ \\
\hline $\mathrm{BP}^{\mathrm{a}}$ & $63.8 \pm 26.8$ & $54.0 \pm 20.0$ & 0.18 & $63.6 \pm 26.6$ & $58.2 \pm 24.4$ & 0.38 \\
\hline $\mathrm{GH}^{\mathrm{a}}$ & $53.6 \pm 14.0$ & $38.1 \pm 13.3$ & 0.0001 & $54.4 \pm 12.6$ & $42.5 \pm 16.8$ & 0.0007 \\
\hline $\mathrm{VT}^{\mathrm{a}}$ & $66.6 \pm 16.1$ & $48.1 \pm 19.5$ & 0.0002 & $68.8 \pm 15.0$ & $50.8 \pm 18.7$ & $<0.0001$ \\
\hline $\mathrm{SF}^{\mathrm{a}}$ & $86.7 \pm 29.3$ & $64.1 \pm 34.4$ & 0.01 & $91.7 \pm 26.8$ & $62.5 \pm 31.6$ & 0.0001 \\
\hline $\mathrm{RE}^{\mathrm{a}}$ & $67.2 \pm 38.1$ & $0 \pm 0$ & $<0.0001$ & $71.7 \pm 36.0$ & $16.7 \pm 33.0$ & $<0.0001$ \\
\hline $\mathrm{MH}^{\mathrm{a}}$ & $62.3 \pm 15.1$ & $49.5 \pm 19.4$ & 0.006 & $62.9 \pm 13.9$ & $53.4 \pm 20.3$ & 0.017 \\
\hline
\end{tabular}

${ }^{\mathrm{a}}$ Mean $\pm \mathrm{SD} .{ }^{\mathrm{b}}$ Number (percentage).

SAS: revised Self-Rating Anxiety Scale; SDS: revised Self-Rating Depression Scale; PCS: physical components summary; MCS: mental components summary; PF: physical functioning; RP: role limitations due to physical problems; RE: role limitations due to emotional problems; SF: social functioning; MH: mental health; VT: energy/vitality; BP: body pain; GH: general health perception.

TABLE 4: Stepwise regression analyses of medical and psychological variables and their relationship to SAS in SLE patients.

\begin{tabular}{lccccr}
\hline SAS & Coef. & Std. Err. & $t$ & $P$ & \\
\hline VT & -0.15 & 0.05 & -3.00 & 0.004 & $-0.25,-0.05$ \\
PF & -0.11 & 0.05 & -2.27 & 0.026 & $-0.22,-0.01$ \\
RP & -0.09 & 0.02 & -3.64 & $<0.001$ & $-0.14,-0.04$ \\
\hline
\end{tabular}

SAS: revised Self-Rating Anxiety Scale; VT: energy/vitality; PF: physical functioning; RP: role limitations due to physical problems. 
TABLE 5: Stepwise regression analyses of medical and psychological variables and their relationship to SDS in SLE patients.

\begin{tabular}{lccccc}
\hline SDS & Coef. & Std. Err. & $t$ & $P$ & \\
\hline SLEDAI & 0.19 & 0.09 & 2.08 & 0.04 & $0.01,0.37$ \\
VT & -0.19 & 0.05 & -3.48 & 0.001 & $-0.30,-0.08$ \\
PF & -0.13 & 0.05 & -2.60 & 0.01 & $-23.8,-0.03$ \\
SF & -0.09 & 0.03 & -2.69 & 0.009 & $-0.15,-0.02$ \\
RE & -0.08 & 0.02 & -3.13 & 0.003 & $-0.12,-0.03$ \\
\hline
\end{tabular}

SDS: revised Self-Rating Depression Scale; SLEDAI: Systemic Lupus Erythematosus Disease Activity Index; VT: energy/vitality; PF: physical functioning; SF: social functioning; RE: role limitations due to emotional problems.

effect on the frequency of anxiety and depression [27]. In the present study, we have found that SLE patients who had low education, unemployed, and low income were prone to anxiety/depression. Female gender and younger age have wellknown associations with depression and confound the SES anxiety/depression relationship in SLE [28]. In the present study, gender was independently associated with depression in SLE. Interestingly, we have found that there were significant correlation between abnormal menstrual history and depression. Whether anxiety/depression is associated with lupus activity remains debatable. Walker SE and colleagues reported that the anxiety severity did correlate positively with SLE activity [29]. Nery et al. reported a significant positive correlation between depression and disease activity [30]. In contrast, other studies reported that there was not relationship between lupus activity and presence of a major depressive episode [31]. In the present study, our group found significant positive correlation between anxiety/depression and disease activity. Health-related quality of life in lupus was found to be significantly worse in comparison with control group. Recent study reported that SLE patients who had significantly poorer health-related quality of life were significantly more depressed and anxious than their healthy counterparts [11]. We have also found the PCS and MCS and all 8 domains of SF-36 except BP were significantly worse than control subjects. The results were similar with the study from Hong Kong. We have also found that the SLEDAI scores were a strong predictor of depression in patients with SLE.

Notably, the results of the present study demonstrated that anxiety in Chinese SLE patients differs from SLE patients in other countries. This could be explained by some cultural features which may influence mental disease diagnosis and management in China. We have found that the prevalence of depression was higher than Hong Kong, and it might be due to cultural differences and SES such as income and medical insurances policy.

In order to identify which variables were most significantly correlated with anxiety and depression, stepwise regression analysis was used. We have found that VT, PF, and RP scales of SF-36 were the most powerful predictors of anxiety of SLE patients. Meanwhile, SLEDAI, VT, PF, SF, and RE domains of SF-36 were significantly accounted for anxiety. It could be explained that impaired quality of life and functional disability were independent risk factors for psychological disorders.
A possible limitation of the present study was that all patients involved in the survey were from only one center and its failure to differentiate between men and women; the gender differences in SLE patients require further analysis in a future study. Another limitation of the study was that we did not detect the impact of proinflammatory cytokines on depression. Recent study reported that higher serum TNF- $\alpha$ level was independently associated with poorer health-related quality of life and more severe depressive symptoms in SLE patients in Singapore [11].

In summary, our study indicated that psychological problems were frequent in Chinese SLE patients. Severe disease status and reduced quality of life significantly correlated with anxiety and depression. Disease activity was higher in anxious and depressed subgroups. Quality of life was decreased in depressed subgroups. Impaired mental health and pain were the most powerful predictors of anxiety and depression. Low SES was independently associated with poor mental health. These findings confirmed the importance of psychosocial interventions in combination with medical therapy for SLE patients.

\section{Authors' Contribution}

Biyu Shen and Wei Tan contribute equally to this work.

\section{Acknowledgments}

The authors want to thank Liren Li, Xinghang Zhu, Genkai Guo, Jie Qian, Haixia Cao, and Yunfei Xia for their assistance with this paper. This work was supported by the National Natural Science Foundation of China (Grant no. 81172841), the Natural Science Foundation of Jiangsu Colleges and Universities Grant no. 09KJB320010, "Top Six Types of Talents" Financial Assistance of Jiangsu Province Grant no. 6, and Jiangsu province's Outstanding Medical Academic Leader Program (LJ201136). This work was also supported by the Science Foundation of Nantong City Grant no. HS2011054, the Beijing Medical Award foundation (FSMYYSNT-001), a project of College graduate research and innovation of Jiangsu Province (CXLX12-0891), the Nantong University Graduate Innovation Program (YKC12037), the Nantong Science and Technology Board (HS12966), and the Bureau of Jiangsu Province (Z2010005), Preventive Medicine Research of Jiangsu province (Y2012083). 


\section{References}

[1] R. H. B. Benedict, J. L. Shucard, R. Zivadinov, and D. W. Shucard, "Neuropsychological impairment in systemic lupus erythematosus: a comparison with multiple sclerosis," Neuropsychology Review, vol. 18, no. 2, pp. 149-166, 2008.

[2] M. Petri, M. Naqibuddin, K. A. Carson et al., "Depression and cognitive impairment in newly diagnosed systemic lupus erythematosus," Journal of Rheumatology, vol. 37, no. 10, pp. 2032-2038, 2010.

[3] G. Marian, E. A. Nica, B. E. Ionescu, and D. G. Carlogea, "Depression as an initial feature of systemic lupus erythematosus? A case report," Journal of Medicine and Life, vol. 3, no. 2, pp. 183-185, 2010.

[4] H. Ainiala, J. Loukkola, J. Peltola, M. Korpela, and A. Hietaharju, "The prevalence of neuropsychiatric syndromes in systemic lupus erythematosus," Neurology, vol. 57, no. 3, pp. 496500, 2001.

[5] Z. S. Meszaros, A. Perl, and S. V. Faraone, "Psychiatric symptoms in systemic lupus erythematosus: a systematic review," Journal of Clinical Psychiatry, vol. 73, no. 7, pp. 993-1001, 2012.

[6] C. M. Greco, T. Li, A. Sattar et al., "Association between depression and vascular disease in systemic lupus erythematosus," Journal of Rheumatology, vol. 39, no. 2, pp. 262-268, 2012.

[7] T. A. Lisitsyna, D. I. Vel'tishchev, O. F. Seravina et al., "Prevalence of mental disorders in SLE patients: correlations with the disease activity and comorbid chronic conditions," Terapevticheskil Arkhiv, vol. 81, no. 6, pp. 10-16, 2009.

[8] L. F. Xie, P. L. Chen, H. F. Pan et al., "Prevalence and correlates of suicidal ideation in SLE inpatients: Chinese experience," Rheumatology International, vol. 32, no. 9, pp. 2707-2714, 2012.

[9] Z. Zakeri, M. Shakiba, B. Narouie, N. Mladkova, M. GhasemiRad, and A. Khosravi, "Prevalence of depression and depressive symptoms in patients with systemic lupus erythematosus: Iranian experience," Rheumatology International, vol. 32, no. 5, pp. 1179-1187, 2012.

[10] C. C. Mok, "Epidemiology and survival of systemic lupus erythematosus in Hong Kong Chinese," Lupus, vol. 20, no. 7, pp. 767-771, 2011.

[11] A. Mak, C. Tang, and R. Ho, "Serum tumour necrosis factoralpha is associated with poor health-related quality of life and depressive symptoms in patients with systemic lupus erythematosus," Lupus, vol. 22, no. 3, pp. 254-261, 2013.

[12] S. Pettersson, M. Lovgren, L. E. Eriksson et al., "An exploration of patient-reported symptoms in systemic lupus erythematosus and the relationship to health-related quality of life," Scandinavian Journal of Rheumatology, vol. 41, no. 5, pp. 383-390, 2012.

[13] T. O. Utset, S. Chohan, S. A. Booth, J. C. Laughlin, M. Kocherginsky, and A. Schmitz, "Correlates of formal work disability in an urban university systemic lupus erythematosus practice," Journal of Rheumatology, vol. 35, no. 6, pp. 1046-1052, 2008.

[14] H.-C. Huang, C.-T. Chou, K.-C. Lin, and Y.-F. C. Chao, "The relationships between disability level, health-promoting lifestyle, and quality of life in outpatients with systemic lupus erythematosus," The Journal of Nursing Research, vol. 15, no. 1, pp. 21-32, 2007.

[15] V. Lorant, D. Deliège, W. Eaton, A. Robert, P. Philippot, and M. Ansseau, "Socioeconomic inequalities in depression: a metaanalysis," American Journal of Epidemiology, vol. 157, no. 2, pp. 98-112, 2003.
[16] S. Stringhini, S. Sabia, M. Shipley et al., "Association of socioeconomic position with health behaviors and mortality," Journal of the American Medical Association, vol. 303, no. 12, pp. 1159$1166,2010$.

[17] L.-S. Tam, A. Wong, V. C. T. Mok et al., "The relationship between neuropsychiatric, clinical, and laboratory variables and quality of life of Chinese patients with systemic lupus erythematosus," Journal of Rheumatology, vol. 35, no. 6, pp. 1038-1045, 2008.

[18] M. C. Hochberg, "Updating the American College of Rheumatology revised criteria for the classification of systemic lupus erythematosus," Arthritis and Rheumatism, vol. 40, no. 9, p. $1725,1997$.

[19] W. W. Zung, "A rating instrument for anxiety disorders," Psychosomatics, vol. 12, no. 6, pp. 371-379, 1971.

[20] W. W. Zung, "A self-rating depression scale," Archives of General Psychiatry, vol. 12, pp. 63-70, 1965.

[21] H. B. M. S. Paro, N. M. O. Morales, C. H. M. Silva et al., "Healthrelated quality of life of medical students," Medical Education, vol. 44, no. 3, pp. 227-235, 2010.

[22] M. Lemstra, C. Neudorf, C. D’Arcy, A. Kunst, L. M. Warren, and N. R. Bennett, "A systematic review of depressed mood and anxiety by SES in youth aged 10-15 years," Canadian Journal of Public Health, vol. 99, no. 2, pp. 125-129, 2008.

[23] R. J. Turner and D. A. Lloyd, "The stress process and the social distribution of depression," Journal of Health and Social Behavior, vol. 40, no. 4, pp. 374-404, 1999.

[24] S. A. Stansfeld, J. Head, and M. G. Marmot, "Explaining social class differences in depression and well-being," Social Psychiatry and Psychiatric Epidemiology, vol. 33, no. 1, pp. 1-9, 1998.

[25] J. P. Mackenbach, A. E. Kunst, A. E. J. M. Cavelaars, F. Groenhof, and J. J. M. Geurts, "Socioeconomic inequalities in morbidity and mortality in western Europe," The Lancet, vol. 349, no. 9066, pp. 1655-1659, 1997.

[26] L. A. Criswell and P. P. Katz, "Relationship of education level to treatment received for rheumatoid arthritis," Journal of Rheumatology, vol. 21, no. 11, pp. 2026-2033, 1994.

[27] A. Waheed, K. Hameed, A. M. Khan, J. A. Syed, and A. I. Mirza, "The burden of anxiety and depression among patients with chronic rheumatologic disorders at a tertiary care hospital clinic in Karachi, Pakistan," Journal of the Pakistan Medical Association, vol. 56, no. 5, pp. 243-247, 2006.

[28] E. A. Bachen, M. A. Chesney, and L. A. Criswell, "Prevalence of mood and anxiety disorders in women with systemic lupus erythematosus," Arthritis Care and Research, vol. 61, no. 6, pp. 822-829, 2009.

[29] S. E. Walker, K. L. Smarr, J. C. Parker et al., "Mood states and disease activity in patients with systemic lupus erythematosus treated with bromocriptine," Lupus, vol. 9, no. 7, pp. 527-533, 2000.

[30] F. G. Nery, E. F. Borba, J. P. Hatch et al., "Major depressive disorder and disease activity in systemic lupus erythematosus," Compr Psychiatry, vol. 48, no. 1, pp. 14-19, 2007.

[31] M. Funauchi, C. Tamaki, T. Yamagata et al., "Quality of life in patients with systemic lupus erythematosus-preliminary survey using a short questionnaire," Japanese Journal of Clinical Immunology, vol. 28, no. 1, pp. 40-47, 2005. 


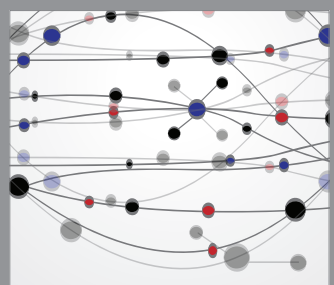

The Scientific World Journal
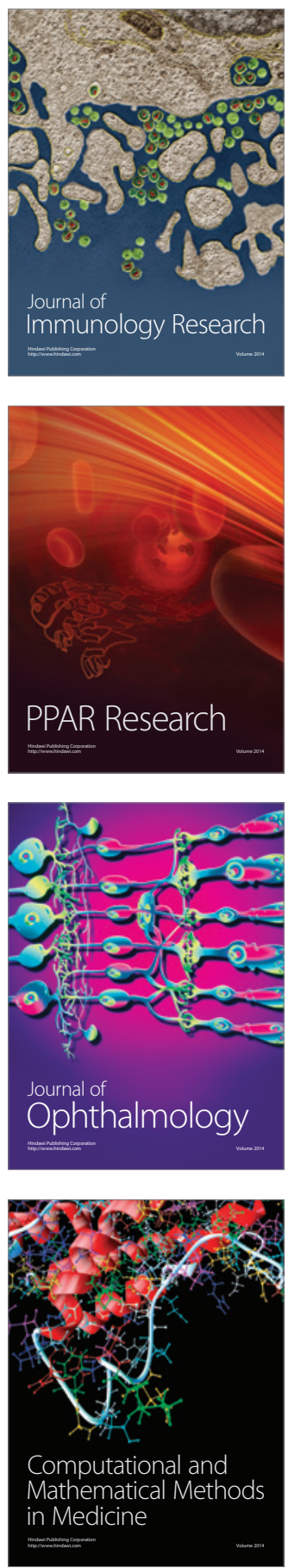

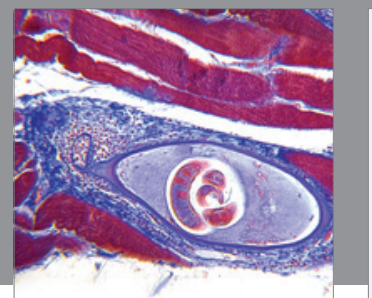

Gastroenterology

Research and Practice
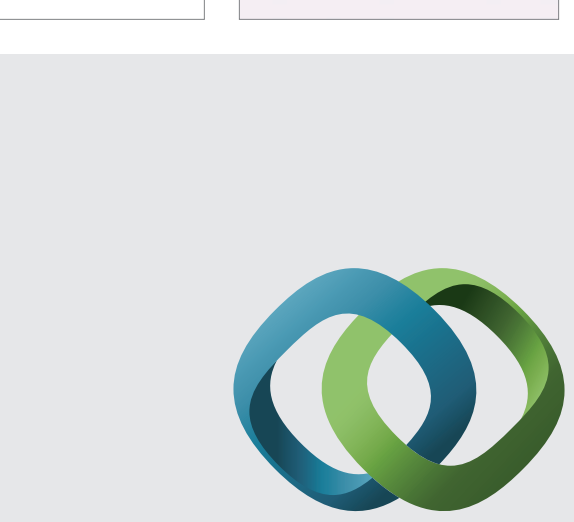

\section{Hindawi}

Submit your manuscripts at

http://www.hindawi.com
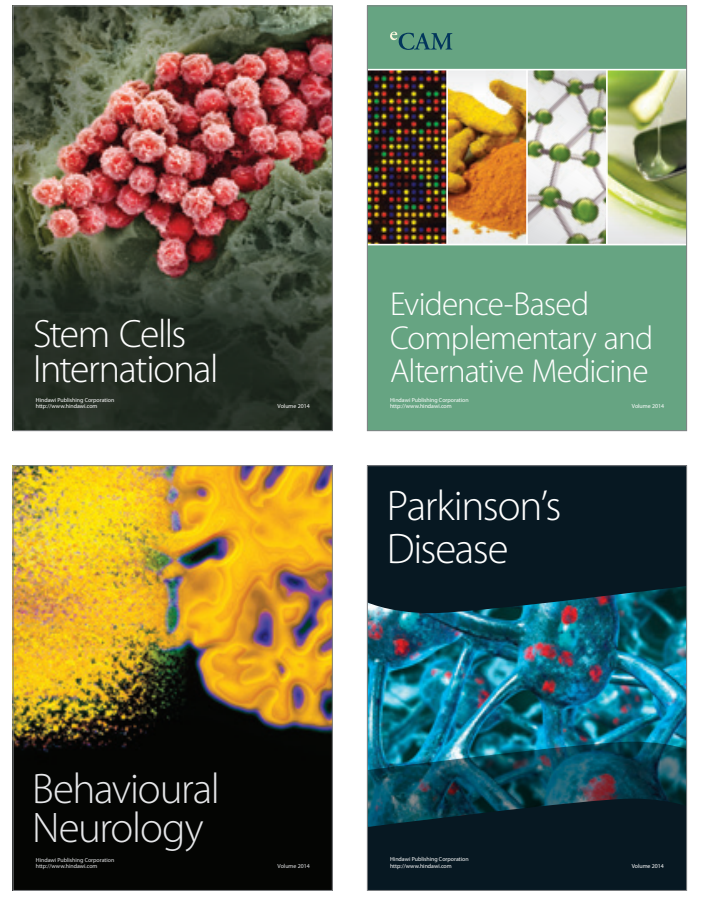
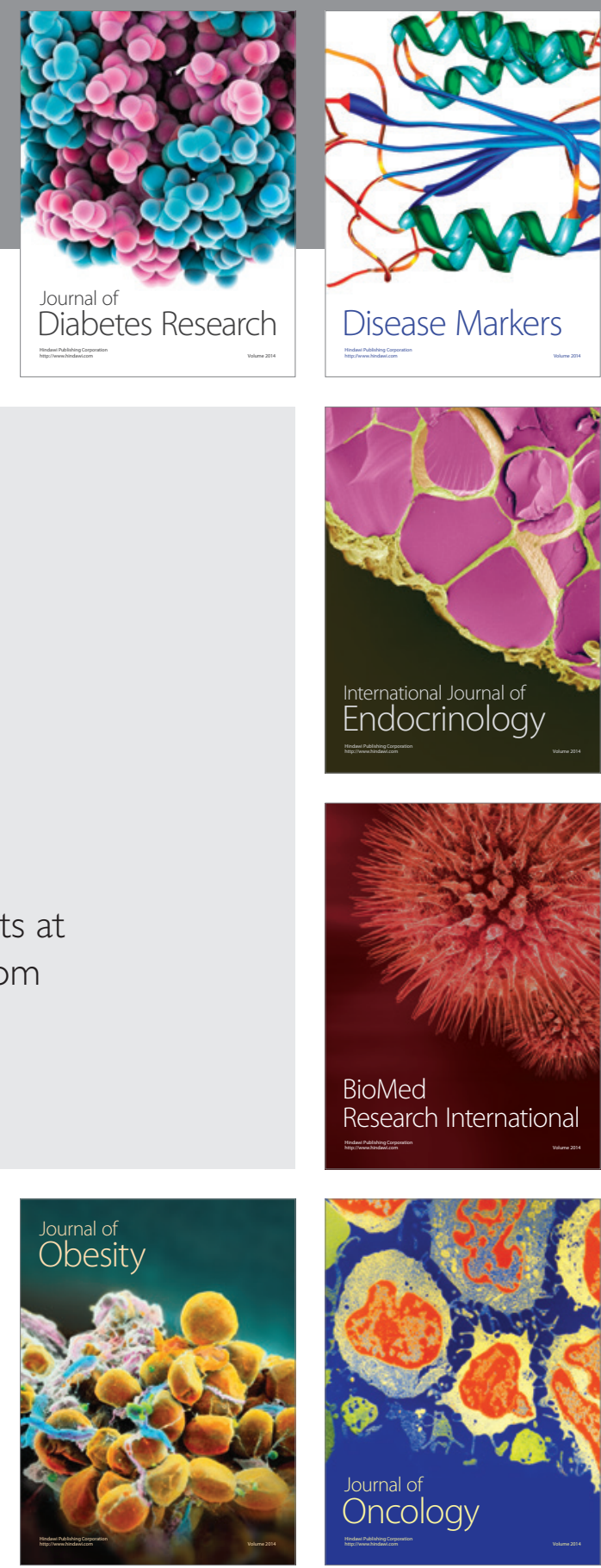

Disease Markers
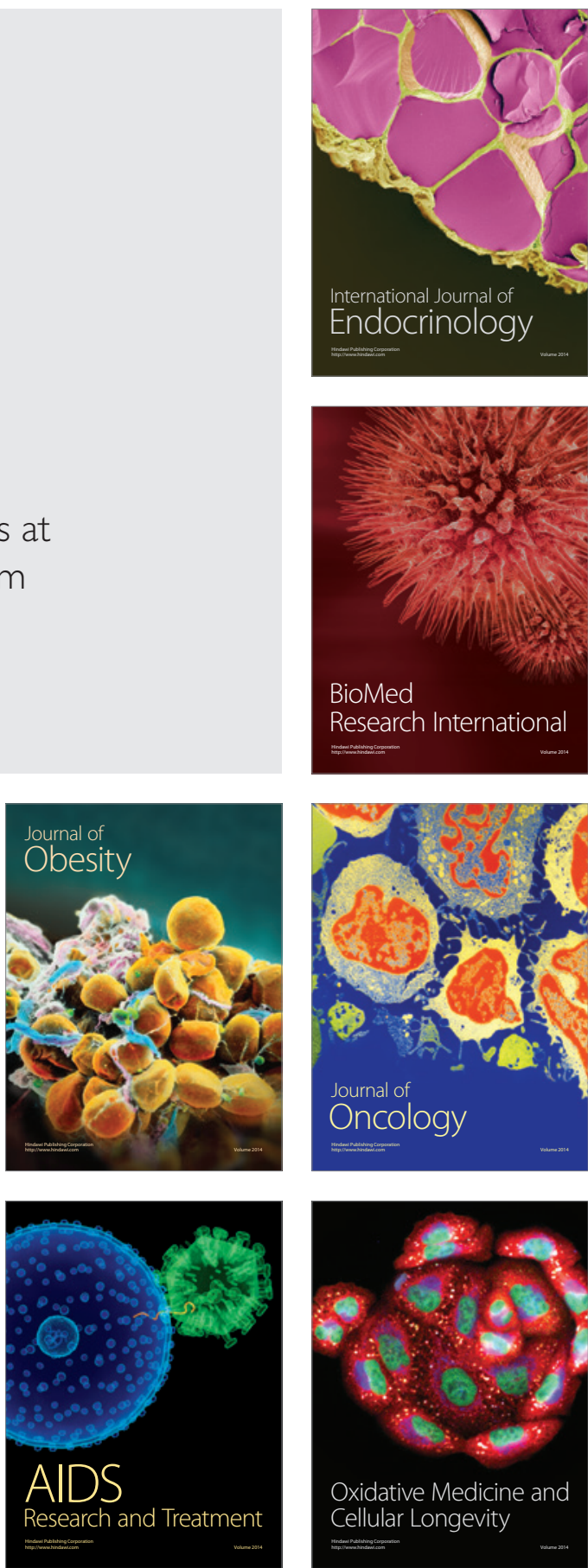